\title{
¿Existe sesgo de género en los estudios de ciencias de la actividad física y el deporte? \\ Is there a gender bias in study programs in sciences of physical activity and sport? \\ *Beatriz Garay Ibañez de Elejalde, **Eider Elcoroaristizabal, *María Teresa Vizcarra Morales, ***Maria Prat Grau, ****Pedrona Serra Payeras, ****Susanna Soler Prat \\ *Universidad del País Vasco (España), ${ }^{* *}$ Gobierno Vasco (España), ***Universidad de Barcelona (España), **** INEFC de Barcelona
}

(España)

Resumen. Con el presente trabajo se desea identificar si existe sesgo de género en los estudios de grado en Ciencias de la Actividad Física y el Deporte en la Universidad del País Vasco (UPV/EHU), para ello, se ha analizado la participación en las pruebas de acceso y las tasas de matrícula de mujeres y hombres, tomando como muestra los datos de estudiantes existentes en los últimos 30 años. Se presenta un estudio descriptivo longitudinal, en el que paralelamente se han analizado las condiciones y normativas que regulan este proceso, con la intención de ver en qué manera inciden éstas en la mayor o menor presencia de las mujeres en dichos estudios. Los datos vertidos indican cuáles son las tendencias de matrícula, así como que este grado está más masculinizado que otros estudios. En la discusión y las conclusiones se muestran las posibles causas de esta situación tomando como referencia la realidad de otras universidades. Como propuesta de futuro se plantean algunas claves para mejorar la orientación que se le da al futuro alumnado antes de elegir sus estudios.

Palabras clave: elección de estudios universitarios; pruebas de acceso; actividad física; deporte; género.

Abstract. This paper aim is to identify gender bias in the studies of the Degree in Physical Activity and Sport Science at the University of the Basque Country (UPV/EHU). Admission tests and enrollment rates of women and men from the last 30 years were used for data analyze. Parallel to this analysis, conditions and regulations governing these processes were analyzed to see to which extent they might affect the presence of women in these studies. The data indicate enrollments trends, as well as that this degree is more masculinized than other studies. The discussion and conclusions show possible causes of this situation taking as a reference the reality of other universities. For future research, we propose some ideas to improve the conditions for future students before choosing their studies.

Keywords: career choice; university studies; admission tests; physical activity; sport; gender.

\section{Introducción}

Este trabajo desea analizar la evolución de la presencia de las mujeres en el Grado de Ciencias de laActividad Física y Deporte(CAFD) de la Comunidad Autónoma Vasca (CAV). Se revisarán las pruebas de acceso, las matrículas y la legislación de sus 30 años de historia. Se han recopilado todos los datos existentes para componer una imagen más completa, ya que no existe ningún estudio previo en la Universidad del País Vasco (UPV/EHU).

En general, la matriculación en la UPV/EHU tiende a equilibrar el número de matrículas de mujeres y hombres. El número de hombres matriculados ha aumentado en titulaciones como Magisterio, Trabajo Social o Enfermería, y también ha aumentado el número de mujeres en los estudios de la Escuela Politécnica y de la Escuela de Ingeniería Técnica de Minas, tradicionalmente masculinizados (Eustat, 2016; Fernández; Hernández \& Rodríguez, 2014). Pero, algunos grados, como el grado de CAFD, mantienen una tendencia mayoritariamente masculina. En investigaciones previas se muestra una menor representación de mujeres en los ámbitos científico-técnicos (Euridyce, 2011; García, Pires \& Fernández, 2013), pero pocas investigaciones centran su atención en CAFD.

El Instituto Vasco de Educación Física (SHEE-IVEF), creado en septiembre de 1986 para impartir la licenciatura en Educación Física, estaba adscrito a la UPV/EHU, con dependencia administrativa del Departamento de Cultura del Gobierno Vasco. En octubre de 2005 se integra en la UPV/EHU cambiando su denominación por la de Facultad de CAFD, coincidiendo con este momento, la licenciatura recibe la denominación de CAFD (Garay, 2005). En 2010, con la entrada en vigor de la LRU, la titulación se adapta al nuevo marco europeo (EEES) y se crea el Grado. En 2016, se reestructuran los centros de la UPV/ EHU, y la Facultad de CAFD, se fusiona con la Escuela Universitaria de Magisterio de Vitoria-Gasteiz dando lugar a la actual Facultad de Educación y Deporte, centro en el que se imparten, además, los grados de Educación Infantil y Primaria con la mención en Educación Física,

Fecha recepción: 20-06-17. Fecha de aceptación: 27-11-17 Beatriz Garay Ibañez de Elejalde beatriz.garai@ehu.eus ofreciendo de este modo, dos grados relacionados con el ámbito de la actividad física.

\section{La presencia de las mujeres en los estudios de CAFD}

Entre los estudios previos destaca el coordinado por Mendizabal (2011) sobre la presencia de mujeres en 24 centros que imparten la titulación de CAFD en España. Los datos están recogidos entre los años 2007 y 2011 y compara el número de matrículas de mujeres y hombres. En el estudio realizado en Galicia por Porto (2009) se analiza las tendencias de matriculación por sexos en el INEF de ACoruña y la Facultad de CAFD de Pontevedra, también los criterios de evaluación, ya que son diferentes para chicas, y chicos. En Cataluña, el estudio realizado por Serra y Soler (2013) sobre los dos centros públicos de estudios de CAFD en Cataluña (Barcelona y Lérida) toma datos entre 1975 y 2012.

EnEuropa destacan los trabajos realizados en Alemania (HartmannTews, 2011) y en Inglaterra (Elliot \& Sander, 2011) que ponen en evidencia el desequilibrio entre matrículas masculinas y femeninas. Se ha constatado también gran preocupación por el carácter androcéntrico de los contenidos del curriculum que suelen estar identificados con el deporte (Fernández, González, Toja \& Carreiro da Costa, 2017; Petry \& Brackenridge, 2001; Serra, Soler, Prat, Vizcarra, Garay \& Flintoff, 2016a; Serra, Vizcarra, Garay, Prat \& Soler, 2016b). La investigación de Dworkin y Messner (2002) estudia cómo son tratados el deporte de competición y el deporte espectáculo, más propios de un curriculum considerado masculino. Las expectativas en torno a la práctica deportiva son de menor calado para las mujeres que para los hombres, ya que se espera menos de ellas en el mundo del deporte(Alvariñas, Fernández \& López, 2009; Leruite, Martos \& Zabala, 2015; Mateos, González, Sánchez, Martínez, Martínez, 2010; Moreno, Martínez \& Alonso, 2006; Pacheco, Soto, Olivárez \& Avila, 2012). La motivación de las mujeres hacia la práctica física se refleja en diversos estudios (Amado, Sánchez-Miguel, Leo, Sánchez-Oliva \& García-Calvo, 2014; Canales \& Martinez, 2018; Elliot \& Sanders, 2011; Flintoff \& Scraton 2001; Jiménez, Cervelló, García, Santos-Ros \& Iglesias, 2007; Martins, Marques, Peralta, Palmeira\& Carreiro da Costa, 2017). En Estados Unidos Davol (2001) analiza las percepciones de las jóvenes hacia la educación 
física para ver qué hay de cierto sobre la apatía de las chicas en las clases de educación física, y llega a la conclusión de que las actividades propuestas, son las que fomentan la no participación de las chicas. Se centra en los contenidos de los programas, en las formas de trabajar y en las experiencias de las chicas adolescentes y en su grado de implicación. Gibbons y Humbert (2008) analizan las clases de educación física de secundaria, y observan que no se tienen en cuenta las necesidades e intereses de las chicas, por lo que proponen cambios para que gocen de las ventajas de involucrarse en clase. Los objetivos del presente estudio son: analizar la evolución de la presencia de las mujeres en las pruebas de acceso y en la matrícula en el grado de CAFD en el País Vasco comparándola con las legislaciones correspondientes; comprender las causas que han podido generar este desequilibrio a partir de la literatura existente; y plantear posibles orientaciones para corregir este sesgo de género.

\section{Método}

Se ha realizado un análisis descriptivo longitudinal de la tendencia en la matriculación en función del sexo del Grado de CAFD de la UPV/ EHU. Se ha analizado la información custodiada en el archivo de la facultad con los datos de matriculación y prematriculación desde su creación, (1986-2016), y se han tenido en cuenta el número de chicas y chicos en:

- $\quad$ Matrículas en las pruebas de acceso.

- $\quad$ Personas superan las pruebas de acceso.

- Matrículas formalizadas.

Se analiza la normativa relacionada con el acceso para ver qué cambios se han ido realizando y en función de qué criterios utilizando el Boletín Oficial del País Vasco entre 1986 y 2016. Se comparan estos datos con los de otros estudios y se realizan propuestas de mejora en función de las causas analizadas.

\section{Resultados}

\section{Personas inscritas en las pruebas de acceso}

El número de personas inscritas en las pruebas físicas de acceso ha ido variando, en los 30 años de existencia se han inscrito un total de 9797 personas, con una media de 326.56 por curso. A partir del curso 2009/ 2010 se superó esa media. Desagregadas estas cifras por sexos, se han inscrito en las pruebas físicas de acceso 7179 hombres y 2618 mujeres. Esto es, se han inscrito por año académico una media de 239.30 hombres (72.82\%) y 87.26 (27.18\%) mujeres.

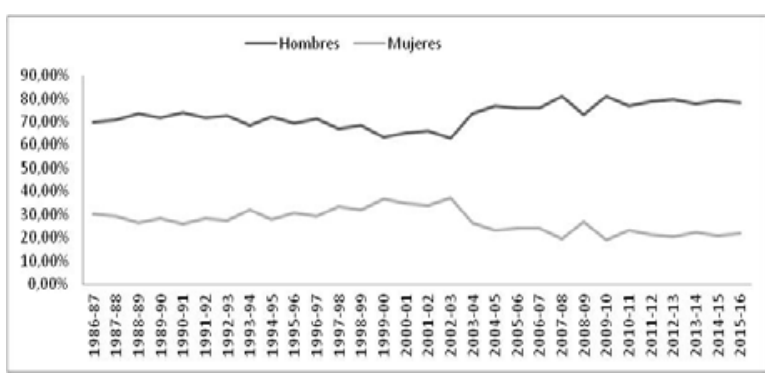

Figura 1. Evolución de las prematrículas a lo largo de los años

El número personas que optan por estos estudios ha aumentado desde el año 2005, año en que el centro se integró en la UPV/EHU pero en este incremento ha sido mayor en el número de hombres. La figura 1 nos muestra la brecha entre la presencia de mujeres y hombres. Entre los años 1997 a 2002 el porcentaje de mujeres que se inscribieron en las pruebas de acceso fue aumentando hasta un 37\%. En el curso 2002/ 2003 esa brecha fue menor, a partir de ese momento y coincidiendo con el cambio de denominación de la licenciatura, se pierde el nombre de educación física, vuelve a disminuir notablemente el número de mujeres, manteniendo esta tendencia hasta el 2015/2016. En los últimos cursos la media de mujeres inscritas ha vuelto a disminuir, oscilando entre un $18.93 \%$ y un $26.29 \%$ del total de las prematriculaciones (tabla 1 ).
Tabla 1.

\begin{tabular}{|c|c|c|}
\hline Curso & Hombres & Mujeres \\
\hline $1986-87$ & $69.67 \%$ & $30.32 \%$ \\
\hline 1987-88 & $70.81 \%$ & $29.18 \%$ \\
\hline $1988-89$ & $73.59 \%$ & $26.40 \%$ \\
\hline 1989-90 & $71.81 \%$ & $28.18 \%$ \\
\hline $1990-91$ & $73.99 \%$ & $26.00 \%$ \\
\hline 1991-92 & $71.58 \%$ & $28.41 \%$ \\
\hline $1992-93$ & $72.45 \%$ & $27.54 \%$ \\
\hline 1993-94 & $68.20 \%$ & $31.79 \%$ \\
\hline $1994-95$ & $75.00 \%$ & $25.00 \%$ \\
\hline $1995-96$ & $69.47 \%$ & $30.00 \%$ \\
\hline $1996-97$ & $71.02 \%$ & $28.98 \%$ \\
\hline $1997-98$ & $66.75 \%$ & $33.24 \%$ \\
\hline 1998-99 & $68.15 \%$ & $31.84 \%$ \\
\hline 1999-00 & $63.39 \%$ & $36.60 \%$ \\
\hline $2000-01$ & $65.29 \%$ & $34.70 \%$ \\
\hline 2001-02 & $75.90 \%$ & $24.09 \%$ \\
\hline 2002-03 & $62.99 \%$ & $37.00 \%$ \\
\hline 2003-04 & $73.70 \%$ & $26.29 \%$ \\
\hline 2004-05 & $76.92 \%$ & $23.07 \%$ \\
\hline 2005-06 & $75.98 \%$ & $24.01 \%$ \\
\hline $2006-07$ & $75.71 \%$ & $24.28 \%$ \\
\hline 2007-08 & $80.78 \%$ & $19.21 \%$ \\
\hline 2008-09 & $73.15 \%$ & $26.84 \%$ \\
\hline 2009-10 & $81.07 \%$ & $18.92 \%$ \\
\hline 2010-11 & $76.74 \%$ & $23.25 \%$ \\
\hline 2011-12 & $78.58 \%$ & $21.41 \%$ \\
\hline $2012-13$ & $79.60 \%$ & $20.39 \%$ \\
\hline 2013-14 & $77.71 \%$ & $22.28 \%$ \\
\hline 2014-15 & $79.27 \%$ & $20.72 \%$ \\
\hline 2015-16 & $78.03 \%$ & $21.96 \%$ \\
\hline Total & $72.82 \%$ & $27.18 \%$ \\
\hline
\end{tabular}

\section{Mujeres y hombres que superan las pruebas físicas}

De 1986 a 2016 han superado las pruebas físicas de acceso 6682 personas. Y la media por curso de personas que ha superado dichas pruebas es de 222.73 (figura 2). Por sexo, superan estas pruebas unos 166.62 hombres aprueban y tan solo 56.50 mujeres por curso condicionado este dato por el menor número de mujeres candidatas, aunque es mayor el número de hombres que acceden a las pruebas, el porcentaje de mujeres que las superan es mayor.

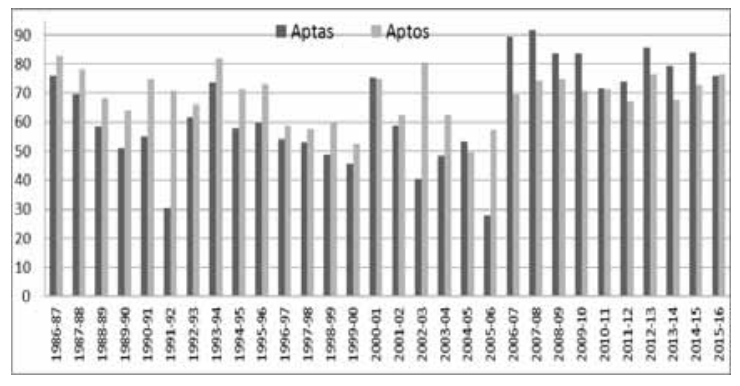

Figura 2. Porcentaje de personas que superan las pruebas de ingreso.

Tabla 2.

\begin{tabular}{|c|c|c|c|c|c|}
\hline Curso & Pruebas físicas & Euskara & $\begin{array}{c}\text { Nota } \\
\text { selectividad }\end{array}$ & $\begin{array}{c}\mathrm{N}^{\circ} \\
\text { pruebas }\end{array}$ & Cambios en el baremo \\
\hline $1986 / 1987$ & $50 \%$ & No & $50 \%$ & 9 & \\
\hline $\begin{array}{l}1987 / 1992 \\
\end{array}$ & $45 \%$ & Si $(10 \%)$ & $45 \%$ & 9 & \\
\hline $\begin{array}{l}1992 / 1993 \\
\end{array}$ & $40 \%$ & Si $(10 \%)$ & $50 \%$ & 7 & \\
\hline 1993/1994 & apto/no apto & Si $(10 \%)$ & $90 \%$ & 6 & $\begin{array}{l}\text { Ajuste baremo, bajan } \\
\text { puntuaciones. }\end{array}$ \\
\hline 1994/1997 & apto/no apto & Si (desempate) & $100 \%$ & 6 & $\begin{array}{c}\text { Sube tiempo mínimo en } \\
\text { prueba velocidad mujeres. }\end{array}$ \\
\hline $\begin{array}{l}1997 / 1998 \\
\end{array}$ & apto/no apto & No & $100 \%$ & 6 & \\
\hline $\begin{array}{l}1998 / 1999 \\
\end{array}$ & apto/no apto & No & $100 \%$ & 6 & \\
\hline 1999/2005 & apto/no apto & No & $100 \%$ & 6 & $\begin{array}{l}\text { Ajuste baremos, } 1 \text { "+ tiempo } \\
\text { mínimo en natación, } 10 \mathrm{~cm} \\
\text { mínimo salto vertical, } 1 \text { "+ } \\
\text { en obstáculos. }\end{array}$ \\
\hline $\begin{array}{l}2005 / 2010 \\
\end{array}$ & apto/no apto & No & $100 \%$ & 6 & \\
\hline $2010 / 2016$ & apto/no apto & No & $100 \%$ & 6 & \\
\hline
\end{tabular}

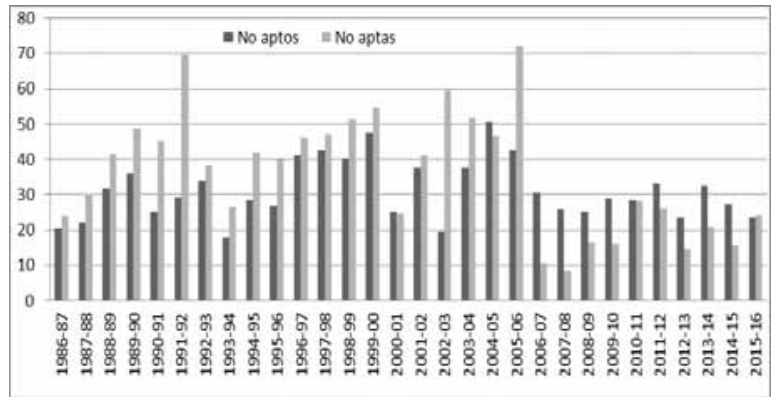

Figura 3. $\mathrm{N}^{\circ}$ personas que no superan pruebas de acceso 
En el año 1992, el número de chicas que aprobó las pruebas de acceso fue muy bajo (24 de las 79 presentadas, un $30 \%$ ), y en vista que la prueba de velocidad puntuaba muy bajo, se adaptaron los baremos aumentando el tiempo mínimo de las chicas (ver figura 3).

En casi todos los cursos el porcentaje de mujeres aprobadas supera el 50\% con respecto a las que se presentan, llegando en el curso 2007/ 2008 incluso al $91.52 \%$. En seis cursos, el porcentaje de mujeres que aprobaron las pruebas fue muy bajo oscilando entre el 27\% y $48 \%$. En los últimos ocho cursos la media de mujeres aprobadas está por encima de la media de personas aprobadas, porcentualmente aprueban menos hombres y más mujeres, siendo esto consecuencia de la revisión de los baremos femeninos para intentar compensar los desequilibrios entre sexos.

El porcentaje de personas aprobadas por sexo en los primeros 14 cursos, esto es, hasta el curso 2000/2001, ha sido mayor en hombres que en mujeres. De manera puntual, en el año académico 2000/2001 el porcentaje de mujeres que aprobaron las pruebas de acceso superó al de hombres, con un $75.37 \%$ frente a un $74.86 \%$.

En los cursos 1991/1992 y 2005/2006, el 30.38\% y el 27.87\% de mujeres superaron las pruebas físicas de acceso. En otros siete cursos menos del 50\% de las mujeres presentadas superaron las pruebas de acceso. Los hombres aprobados, sólo han bajado del $50 \%$ en el curso 2004/2005 con un $49.50 \%$.

\section{Evolución de los requisitos de acceso en el Grado de CAFD}

Los requisitos de acceso a los estudios de Grado han ido variando. En el curso 1986/1987 las pruebas físicas de acceso y la nota de la prueba de acceso a la Universidad valían el 50\% cada una. Estos requisitos se mantuvieron hasta el curso 1991/1992. En este momento las pruebas físicas pasan a tener menor valor, $45 \%$ sobre la nota final, la nota de euskara un 10\% y la de Selectividad 45\%. En 1992/1993, se bajó el valor de la pruebas físicas de un $45 \%$ a un $40 \%$, subiendo el valor de la nota de selectividad al 50\% y manteniendo el valor de la prueba de euskara. En 1993/1994 las pruebas físicas pasaron a ser un requisito mínimo para el acceso a los estudios, teniendo la valoración de apto o no apto, mientras que la selectividad pasó a tener más peso, un 100\% sobre la nota final entre las personas aptas (tabla 2).

Se ha analizado el valor específico otorgado a cada prueba de acceso, y el número y tipo de pruebas físicas realizadas en cada curso. Los primeros siete años se realizaron nueve pruebas de acceso. En el año 1992 se realizaron siete pruebas, y de 1993 en adelante se han realizado seis pruebas.

En la tabla 3 se puede ver la evolución de las pruebas físicas y el tipo de pruebas centradas en la condición física. Durante los seis primeros años se realizó una prueba de flexibilidad, que fue descartada a partir de 1992, siendo en esta prueba donde mejor puntuaban las mujeres. En el curso 2006/2007 se hizo una revisión del baremo femenino ya que se detectó un aumento considerable en el número de chicos que superaban las pruebas.

\begin{tabular}{|c|c|c|c|}
\hline $1986 / 1987$ & $1988 / 1991$ & $1992 / 1993$ & $1994 / 2016$ \\
\hline Prueba natación & Prueba natación & Prueba natación & Prueba natación \\
\hline Salto vertical & Salto vertical & Salto vertical & Salto vertical \\
\hline Carrera de obstáculos & Carrera de obstáculos & Carrera de obstáculos & $\begin{array}{l}\text { Carrera de } \\
\text { obstáculos }\end{array}$ \\
\hline $\begin{array}{l}\text { Lanzamiento balón } \\
\text { medicinal }\end{array}$ & $\begin{array}{l}\text { Lanzamiento balón } \\
\text { medicinal }\end{array}$ & $\begin{array}{l}\text { Lanzamiento balón } \\
\text { medicinal }\end{array}$ & $\begin{array}{l}\text { Lanzamiento balón } \\
\text { medicinal }\end{array}$ \\
\hline $50 \mathrm{~m}$ lisos & $50 \mathrm{~m}$ lisos & $50 \mathrm{~m}$. lisos & Prueba de velocidad \\
\hline $\begin{array}{l}\text { Resistencia: } \\
2000 \mathrm{~m}(\mathrm{H}) \text { y } 1000 \mathrm{~m}(\mathrm{M})\end{array}$ & $\begin{array}{l}\text { Resistencia: Course } \\
\text { Navette }\end{array}$ & $\begin{array}{l}\text { Resistencia: Course } \\
\text { Navette }\end{array}$ & $\begin{array}{l}\text { Resistencia: Course } \\
\text { Navette }\end{array}$ \\
\hline Abdominales & $\begin{array}{l}\text { Prueba de adaptación } \\
\text { balón }\end{array}$ & $\begin{array}{l}\text { Prueba de adaptación al } \\
\text { balón }\end{array}$ & \\
\hline Flexión de brazos & Flexión de brazos & & \\
\hline $\begin{array}{l}\text { Flexión profunda del } \\
\text { cuerpo (flexibilidad) }\end{array}$ & $\begin{array}{l}\text { Flexión profunda del } \\
\text { cuerpo (flexibilidad) }\end{array}$ & & \\
\hline
\end{tabular}

Tras los ajustes de los baremos, el porcentaje relativo de mujeres aprobadas aumentó de forma notable, superando las pruebas físicas el $89.47 \%$ de mujeres y el $70.82 \%$ de hombres presentados. A partir de esta fecha esta tendencia se ha mantenido.

La figura 4 muestra el porcentaje de personas que superan las pruebas físicas y de quienes se matriculan una vez aplicada la nota de
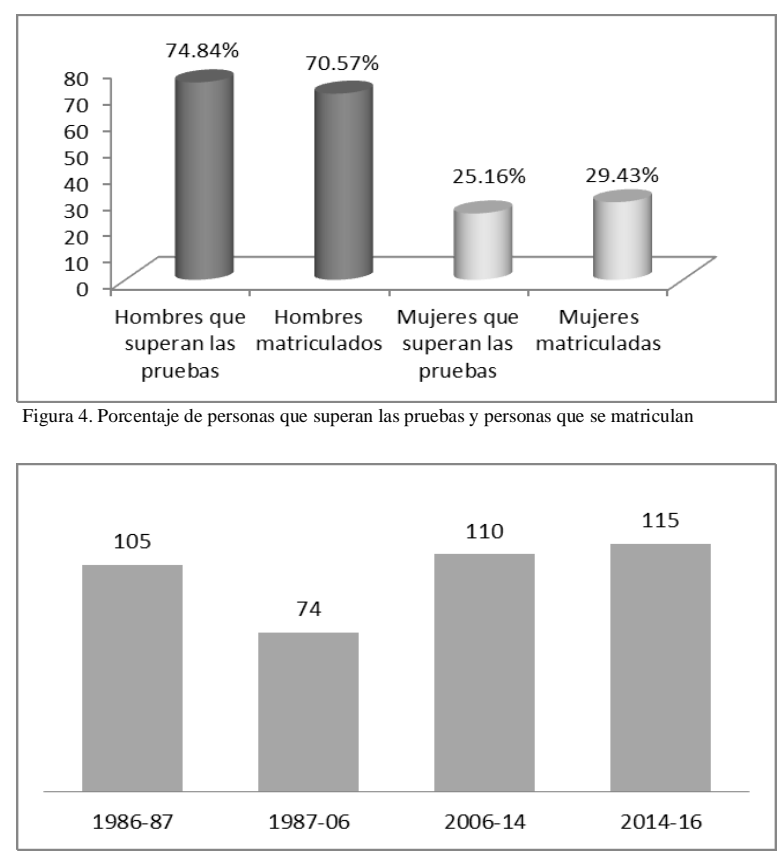

Figura 5. Evolución del $\mathrm{n}^{\circ}$ de plazas ofertadas

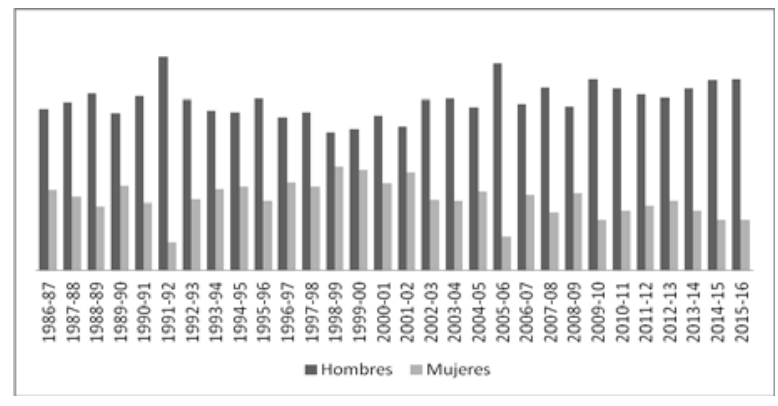

Figura 6. $\mathrm{N}^{\circ}$ alumnas y alumnos matriculados en cada curso

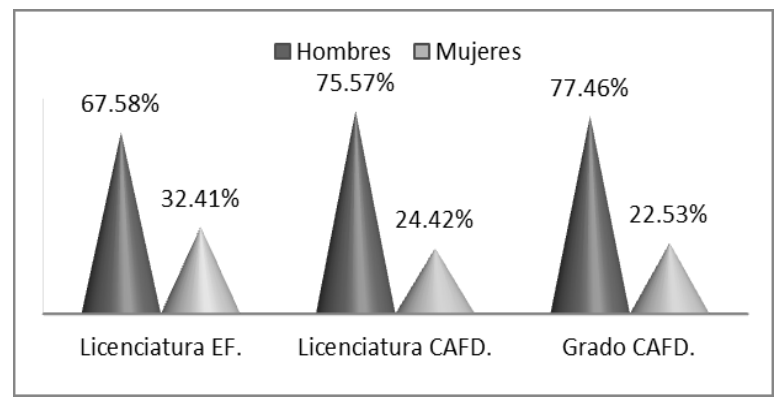

Figura 7. Porcentaje de personas matriculadas en diferentes etapas de la facultad

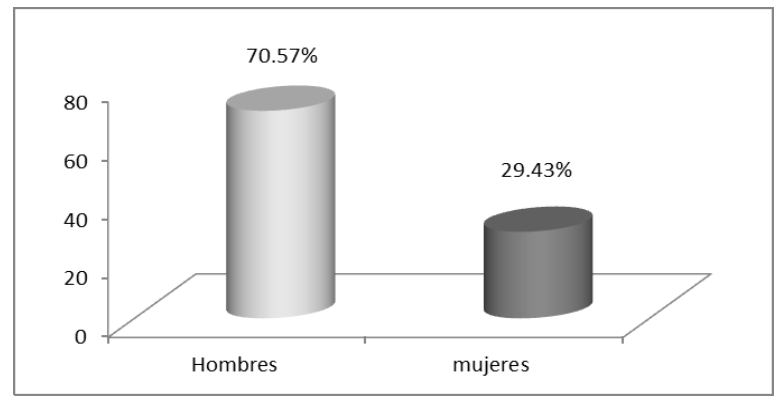

Figura 8. Media de alumnos y alumnas matriculados desde 1986 hasta 2016

selectividad, parece que las notas de selectividad de las mujeres son mejores que las de los hombres. Para entender mejor la figura 4, se va a aportar un ejemplo, en el curso 2008/2009 se inscriben 298 personas (de ellas el $73.15 \%$ son chicos y el $26.85 \%$ chicas), superan las pruebas 230 personas (163 chicos, $70.83 \%$ y 67 chicas, $29.13 \%$ ) y consiguen matricularse 100 personas de ellas 68 son chicos (68\%) y 32 chicas (32\%). 
En los cuatro primeros cursos estudiados se daba mayor paridad entre quienes se inscribían en las pruebas físicas de acceso y quienes lograron matricularse. En el curso 1991/1992 tan solo el 10.12\% de las mujeres inscritas en las pruebas físicas logró matricularse. Desde el curso 1992/1993 se observó un cambio de tendencia. En los últimos diez cursos, desde el 2006/2007, el porcentaje de mujeres inscritas que logran matricularse supera al porcentaje de los hombres que superan las pruebas.

Teniendo en cuenta que el total de personas inscritas a las pruebas físicas de acceso por término medio es 327.80 personas, y solo consiguen matricularse un $27.84 \%$ (88.43 personas) parece un dato positivo que sean las chicas las que una vez inscritas consigan en mayor número que los chicos entrar en el grado de CAFD. Se inscriben $27.18 \%$ de chicas y $72.82 \%$ de chicos, por término medio, y consiguen matricularse un $29.43 \%$ de chicas y $70.57 \%$ de chicos.

\section{Número de plazas ofertadas en los estudios de CAFD}

En el curso 1987/1988 se ofertaban 74 plazas mientras hoy en día la oferta ha aumentado hasta las 115 plazas. El aumento en el número de plazas ofertadas se produjo con la integración de la facultad en la UPV/ EHU, a partir del curso 2006/2007 (figura 5).

El aumento del número de matrículas no es proporcional para ambos sexos, es mayor el aumento en hombres. Los resultados de los dos últimos cursos no superan los datos de matriculación que hubo en los cursos 1999/2000 y 2000/2001 (figura 6).

A partir del curso 1993/1994 en el que el 100\% de la nota estaba relacionada con la nota de Selectividad se observó un aumento del número de mujeres que consiguieron matricularse. Los datos de matrículas por sexo en CAFD son concluyentes, el número medio de hombres matriculados por curso dobla la media de mujeres en el primer período (1986-2005), cuando se obtenía la Licenciatura en Educación Física, en el segundo período (2005-2010) en el que se imparte la Licenciatura en CAFD se triplica este porcentaje, y en el último período (2010-2016) de adaptación al EEES, Grado de CAFD el porcentaje de chicas que cursan estos estudios no supera el 30\% (figura 7).

En los 30 años de vida de estos estudios, el número de mujeres matriculadas no supera en ninguna etapa el tercio del total (figura 8). El descenso progresivo de las matrículas femeninas es preocupante.

\section{Discusión y conclusiones}

Los datos recogidos evidencian que existe una fuertemasculinización en el grado de CAFD en la CAV, y así, tan solo se matriculan una media de 29.43\% mujeres, fenómeno que ha sido observado también en investigaciones realizadas en otros contextos (Mendizabal, 2011; Porto, 2009; Serra \& Soler, 2013). La presencia de mujeres en los estudios de CAFD de la UPV/EHU se ve menos reforzada en dos momentos, en el año 2005 cuando se cambia la denominación de Licenciatura de Educación Física a Licenciatura de CAFD, y el segundo momento es en 2010, cuando se cambia de Licenciatura a Grado. Mientras la licenciatura mantuvo el nombre de Educación Física la tendencia de matriculación femenina fue aumentando hasta llegar a un 37\% en el año 2002. En los últimos años, que no aparece el término Educación Física, el porcentaje de mujeres ha bajado considerablemente, y no se ha llegado a superar nunca el 26\% de mujeres, llegando incluso al 18\% en el año 2009. En los datos presentados por Mendizabal (2011) el número de mujeres matriculadas no era superior a un 30\% en ninguno de los 24 centros estudiados entre los años 2007 y 2011. Los trabajos realizados en Europa sobre este tema también coinciden con estos datos, aunque el porcentaje de mujeres matriculadas es ligeramente mayor en Alemania un 33.8\% (Hartmann-Tews, 2011) y en Inglaterra un 32\% (Elliot \& Sander, 2011).

Algo parecido ocurre con el cambio de denominación del centro, en el curso 2005/2006, el centro pasa de ser Instituto Vasco de Educación Física a Facultad de Ciencias de Actividad Física y Deporte y se vuelve a producir un descenso de mujeres, al igual que sucede en otras comunidades autónomas (Areces, et al., 2011; Porto, 2009; Prat, Carbonero \& Soler, 2013). Cada vez que el término «educación» desaparece, ya sea del título universitario o del centro que lo imparte, hay una caída del número de mujeres que se interesan por los estudios y un incremento de hombres. Así, según Davol (2001), esta desaparición de la palabra Educación Física, puede ser una de las razones del menor acceso de las mujeres, ya que en sus experiencias deportivas no suele recibir la misma valoración que las de los hombres. Parece que al percibir una orientación más tendente al rendimiento, el número de mujeres disminuye. En la misma línea Petry y Brackenridge (2001) destacaron el carácter androcéntrico del curriculum. El que estos estudios se identifiquen con el deporte de competición y el deporte espectáculo, les imprime un carácter más androcéntrico (Dworkin\& Messner, 2002), siendo más difícil que las mujeres elijan estos estudios. Así mismo, las expectativas sociales en función del sexo hacen que se esperen comportamientos diferentes de cada sexo (Moreno, et al., 2006) y dificultan que las mujeres elijan este tipo de estudios. La diferenciación de roles que la sociedad dibuja para cada sexo, espera de los hombres unas prácticas de fuerza o riesgo, y de las mujeres unas prácticas relacionadas con el ritmo, y la expresión (Alvariñas, et al. 2009). Además, las pruebas de acceso suelen estar asociadas al modelo deportivo masculino.

La normativa sobre las pruebas de acceso va ligada a la presencia de mujeres, así cuando la nota de Selectividad comienza a tener más peso en la nota total para el acceso, se incrementa la matrícula de mujeres hasta el 31.79\%, y se mantiene hasta el año 2001 (35\%). Desde el año 2003 no se ha conseguido volver a superar el 26\%. En los dos grados relacionados con el ámbito de la actividad física en la UPV/EHU, el número de chicos es mayor que el de las chicas. Varios trabajos señalan que este desinterés se relaciona con la competición en unos deportes hegemónicos concretos, tradicionalmentemasculinos (Davol, 2001; Elliot \& Sanders, 2011). El número de mujeres implicadas en prácticas físicas fuera del contexto escolar, fundamentalmente en actividades como aerobic, natación o andar a pie van en aumento (Flintoff \& Scraton, 2001), en el estado español el $77 \%$ de chicos y el 66\% de chicas realizan actividad física no organizada (Prat, et al., 2013). Y sin embargo, el profesorado de educación física, tanto en los grados, como en los centros escolares, sigue señalando el desinterés de las chicas por la actividad física. En el caso estudiado el porcentaje relativo de mujeres que aprueban las pruebas físicas es algo mayor que el porcentaje relativo de hombres del $100 \%$ de hombres tan solo aprueban las pruebas físicas el $27.78 \%$ y del $100 \%$ de chicas un $31.59 \%$. No parece que haya una diferencia muy grande, por lo tanto, esta razón de la participación deportiva, no puede ser determinante a la hora de explicar porque las chicas no eligen los estudios de CAFD en un porcentaje similar al de los chicos. Además aducen que la falta de modelos de referencia femeninos entre el profesorado de estos estudios.

Se puede intuir que en el comportamiento en las matrículas de la facultad estudiada, existen tres ejes que se repiten al explicar la masculinización estructural en los estudios de CAFD: los procesos de dualización de género, un modelo de educación física androcéntrica centrada en el deporte y alejada de los intereses de las chicas y la existencia de una cultura femenina que no se recoge en las clases de EF ni en los estudios de CAFD (Serra, et al, 2016a).

Entre las propuestas planteadas para superar las barreras que persisten en la masculinización de los estudios de ciencias del deporte la idea que se repite en los estudios revisados, es plantear cambios en el curriculum (Petry \& Brackenridge, 2001).

\section{Prospección de futuro}

Sería necesario explicar y concretar mejor la oferta de los estudios de CAFD en la página web de la universidad, y en las ferias de orientación, para aclarar mejor cuál es el fin de estos estudios.

Para incrementar la presencia de las chicas se debería incidir más en aquellas opciones que son más atractivas para ellas, como son la salud, estudios sobre aspectos sociales, y la educación física escolar tal como recomiendan los estudios revisados (Areces, et al., 2011; HartmannTews, 2011; Petry \& Brackenridge, 2001; Porto, 2009). En la denominación de los estudios debería incluirse el término «educación» en lugar 
de deporte (Elliot \& Sanders, 2011).

Habría que deconstruir la lógica binaria con que se perciben la diferenciación sexual, y ofrecer nuevas construcciones sociales que tengan sentido para todas las personas y contribuir a establecer condiciones de vida más justas y equitativas (Gallo \& Martínez, 2015). Se necesita realizar una reflexión profunda en torno a la aceptación acrítica de los estereotipos profesionales del deporte, ya que tiene consecuencias negativas en el pensamiento de sus estudiantes.

Queda pendiente un debate sobre la pertinencia que tienen las pruebas de acceso y sobre la tipología de las pruebas de acceso, solicitando unas pruebas no excluyentes.

Puede ser que debido a las expectativas profesionales las chicas se inclinen por otro tipo de estudios porque piensan que otras opciones les facilitarán la inserción laboral y les llevarán a conseguir empleos mejor retribuidos para ellas (Kirk \& Oliver, 2014).

Se debería investigar sobre los modelos deportivos femeninos que se utilizan y conocer la cantidad y la calidad de los modelos que se utilizan en la formación inicial, ya que a las chicas y a las niñas les faltan imágenes de mujeres que sean populares y triunfadoras (Delfy, 1998).

\section{Referencias}

Alvariñas, M., Fernández, M.A., \& López, C. (2009). Actividad física y percepciones sobre deporte y género. Revista de Investigación en Educación, 6, 113-122.

Amado, D., Sánchez-Miguel, P.A., Leo, F.M., Sánchez-Oliva, D., \& GarcíaCalvo, T. (2014) Diferencias de género en la motivación y percepción de utilidad del deporte escolar / Gender differences in motivation and perception of utility of the scholar sport. Revista Internacional de Medicina y Ciencias de la Actividad Física y el Deporte, 14 (56), 651-664. Http://cdeporte.rediris.es/revista/revista56/ artdiferencias510.htm

Areces, A., Mosquera, M., \& Martin, R. (2011). 25 Anos da Faculta de Ciencias do deporte e a Educaión Física/INEF de Galicia (19862011). A Coruña: Universidade da Coruña.

Canales, I, \& Martinez, A. (2018). La ineficacia motriz en la educación física: perspectiva femenina. Retos. Nuevas tendencias en Educación Física, Deporte y Recreación, 33, 81-84.

Davol, D.L. (2001). Female student's apathy toward secondary physical education. (Thesis). University of Georgia: Athens, Georgia.

Delfy, L.A. (1998). Career opportunity in sport. Journal of Physical Education, Recreation \& Dance, 69 (7), 17-22.

Dworkin, S., \& Messner, M. (2002). Gender relations and sport. Sociological Perspectives, 45 (4), 347-352.

Elliot, D., \& Sander, L. (2011). Why females don't do sport degrees. Journal of Hospitality, Leisure, Sport \& Tourism Education, 10 (1), 85-98.

EURYDICE (2011). Diferencias de género en los resultados educativos: medidas adoptadas y situación actual en Europa. Madrid: Ministerio de Educación.

EUSTAT Instituto Vasco de Estadística. (2016). Alumnado matriculado en las universidades de la C.A.V. en estudios de primer y Segundo ciclo. Recuperado de http://www.eustat.eus/bankupx/pxweb/es/spanish/ -/PX_2320_sup01.px/table/tableViewLayout2/?rxid=e8ce24e6e93d-4cab-86ba-c6f23587bf86\#axzz4WFUFgugQ

Fernández, C., Hernández, J., \& Rodríguez, S. (2014). Género y preferencias profesionales en universitarios de estudios científico- tecnológicos. Revista Española de Orientación Psicopedagogica, 25(1), 7893.

Fernández, M., González, M., Toja, B., \& Carreiro da Costa, F. (2017). Valoración de la escuela y la Educación Física y su relación con la práctica de actividad física de los escolares. Retos. Nuevas tendencias en Educación Física, Deporte y Recreación, 31, 312-315

Flintoff, A., \& Scraton, S. (2001). Stepping into active leisure? Young women's perceptions of active lifestyles and their experiences of school physical education. Sport, Education and Society, 6(1), 5-21.

Jiménez, R, Cervelló, E, García, T, Santos-Rosa, F.J., \& Iglesias, D (2007). El género como variable moduladora de la orientación disposicional, percepción del clima motivacional, percepción de igualdad de trato y comportamientos de disciplina de los discentes en las clases de educación física. Revista Iberoamericana de Psicología del Ejercicio y el Deporte, 2(1), 13-23.

Garay, B. (2005). Situación actual y tendencias en Pedagogía de la Actividad Física y el Deporte, 241-263. En: González Suárez, A. (coord.)
Avances de Ciencias del Deporte, Bilbao: UPV/EHU.

Gallo, L.E., \& Martínez, L.J. (2015). Líneas pedagógicas para una educación corporal. Cadernos de pesquisa, 147 (45), 612-629. http:// dx.doi.org/10. 1590/198053143215

García, J., Pires, F., \& Fernández, J. (2013). Preferencias profesionales de los estudiantes de licenciatura en Ciencias de la Actividad Física y el Deporte de la Universidad de Sevilla. Retos. Nuevas tendencias en Educación Física, Deporte y Recreación, 23, 39-42.

Gibbons, S., \& Humbert, L. (2008). What are middle-school girls looking for in physical education? Canadian Journal of Education, 31(1), 167-186.

Hartmann-Tews, I. (2011). Gender issues at the German Sport University. Grup de’Investigació Social I Educativa en l'Activitat Física I’Esport. Recuperado de inefcgiseafe.files.wordpress.com/.../prof-dr-Hartmanntews-Gender issues at the German Sport university. pdf.

Kirk, D., \& Oliver, K. L. (2014). La misma historia de siempre: reproducción y reciclaje del discurso dominante en la investigación sobre la educación física de las chicas. Apunts. Educación Física y Deportes, 116(2), 7-22

Leruite, M.T., Martos, P., \& Zabala, M. (2015). Análisis del deporte femenino español de competición desde la perspectiva de protagonistas clave. Retos. Nuevas tendencias en Educación Física, Deporte y Recreación, 28, 3-8.

Martins, J., Marques, A., Peralta, M., Palmeira, A., \& Carreiro da Costa, F. (2017). Correlates of physical activity in young people: A narrative review of reviews. Implications for physical education based on a socio-ecological approach. Retos, Nuevas tendencias en Educación Física, Deporte y Recreación, 31, 292-299.

Mateos, C, González, A, Sánchez, A, Martínez, A., \&Martínez, M.J. (2010). An approach to the historical development of female athletics in the Olympic Games. Journal of Human Sport \& Exercise, 5 (2) 2, 117-126.

Mendizabal, S. (2011). Deporte y mujer. Cátedra Ciudad 2011: Deporte, Universidad y Ciudad. Valencia: Universidad de Valencia. Recuperado de http://inndeavalencia.com/wp-content/uploads/2012/05/ 05_Catedra_ciudad_2011_Deporte_universidad_ciudad/15.pdf

Moreno, J.A., Martínez, C., \& Alonso, N. (2006). Actitudes hacia la práctica físico-deportiva según el sexo del practicante. Revista Internacional de Ciencias del Deporte, 3(2), 20-43.

Petry, K., \& Brackenridge, C. (2001). Women's Careers in Sport and Leisure Sciences/ Studies in Germany: Comparison with the English Situation. International Council for Health, Physical Education, Recreation, Sport and Dance, 37(3), 25-28.

Porto, B. (2009). Feminización y masculinización en los estudios de maestro y educación física en Galicia. Revista de Investigación en Educación, 6, 50-57.

Prat, M., Carbonero, L., \& Soler, S. (2013). La perspectiva de género en los planes de estudio del grado de CAFD en Cataluña. Feminismo/s, 21, 117-137.

Pacheco, L.A, Soto, F, Olivárez, A., \& Avila, M. (2012). Motivational factors related to female participation in collegiate sports. Journal of Human Sport and Exercise, 7 (4), 783-793.

Ramírez, G., Piedra, J., Ries, F., \& Rodríguez, A.R. (2014). La mujer y el deporte en el cine del siglo XXI (2000-2009). Revista Internacional de Medicina y Ciencias de la Actividad Física y el Deporte, 14 (56), 719-734. Http://cdeporte.rediris.es/revista/revista56/ artABFR506.htm

Serra, P., \& Soler, S. (2013). Evolución de la presencia de mujeres en los estudios de CAFD en el INEF de Cataluña: Datos para la reflexión. En J. Aldaz, A. Dorado, P.J., Jiménez \& A. Villanova (eds.) Responsabilidad social, ética y deporte, 411-419. Libro de Actas del XII Congreso de AEISAD 2012. Investigación Social y Deporte, 11. Madrid: Ibersaf industrial.

Serra, P., Soler, S., Prat, M., Vizcarra, M.T., Garay, B., \& Flintoff, A. (2016a). The (in) visibility of gender knowledge in the Physical Activity and Sport Science Degree in Spain. Sport, Education and Society. http://dx.doi.org/10.1080/13573322.2016.1199016.

Serra, P., Vizcarra, M. T., Garay, B., Prat, M., \& Soler, S. (2016b). Análisis del discurso de género en las matrices curriculares de la Educación Física y el Deporte. Movimento, 22 (3), 821-834. http://dx.doi.org/ 10.1080/13573322.2016.1199016.

Universidad del País Vasco - UPV/EHU (2011). Mujeres y hombres en la UPV/EHU 2009- 2010. II Diagnóstico de la dirección para la igualdad de la UPV/EHU. Recuperado de http://www.berdintasuna.ehu.es/ p 234 - con t ent/e s/contenidos/informacion/ planigualdad_informe_situacion/es_2011/informe.html. 